\title{
Visual loss: a rare presentation of mitral valve stenosis
}

\author{
B.U.D. Naeem ${ }^{1}$, A. Masood ${ }^{1}$, A. Hannan ${ }^{2}$ \\ 1 Fauji Foundation Hospital, Rawalpindi, Pakistan \\ 2 Al-Shifa Trust Eye Hospital, Rawalpindi, Pakistan
}

Keywords: Retina; embolus; mitral valve stenosis; branch retinal artery occlusion; ischaemia

\section{Abstract}

We present a case of sudden loss of vision in left eye due to occlusion of the infero-temporal branch of the retinal artery. The patient had no previous history of cardiac disease. Cardiologist's review revealed the diagnosis of severe mitral valve stenosis as a source of embolism. This is a very rare presentation of mitral valve stenosis which usually presents with pulmonary symptoms and stroke. Mitral stenosis must be considered as an important differential diagnosis in any thromboembolic phenomenon including retinal arterial occlusion.

\section{Introduction}

Mitral valve stenosis is a common valvular heart disease. Rheumatic fever is still the most common cause of mitral stenosis with its symptoms appearing clinically years after acute infection. Mitral valve stenosis presents clinically with a wide array of presentations. Two of the most common clinical presentations are caused by increased pulmonary vascular resistance and due to showering of emboli from the defective valve which causes thromboembolic phenomenon [1]. Mitral valve stenosis is thrombogenic even if the patient is in sinus rhythm [2]. Thus peripheral embolism is a major concern in such patients. Ultimate destination of this embolism is mostly the brain or sometimes the myocardium [3]. Apart from these two tissues, the retina is also a rare location of the embolus to lodge. The presentation of retinal occlusive disease with the ultimate origin of the embolus from the cardiac valve is only mentioned in case reports [4-7]. With that being said, it is even rarer for mitral valve disease to present for the first time as a retinal occlusive disease [8]. We

Correspondence: Dr. Abdul Hannan

E-mail: shhannan@hotmail.com present here such a unique case of severe mitral valve stenosis presenting with visual loss complains due to branch retinal artery occlusion.

\section{Case Report}

We present a case of a 44 year old female with no previous history of cardiac disease presenting to the eye department with painless, sudden, visual loss in the left eye for more than 48 hours. Patient was normotensive and normoglycemic with no complaints of chest pain, dyspnoea or orthopnoea. She had a visual acuity of hand movements in the left eye while 6/9 on snellens chart in the other eye. Her intra-ocular pressures in both eyes were within the normal range. The left eye pupil had marked relative afferent pupillary defect. On fundal examination she was found to have left infero-temporal branch retinal artery occlusion. The artery was occluded at its origin in the optic disc with a non-refractile fibrinoplatelet embolus visible, but not moving on ocular massaging. Patient was given intra-venous mannitol $5 \mathrm{mg} / \mathrm{kg}$ body weight to lower intra-ocular pressure in an attempt to dislodge the embolus. YAG-Laser embolectomy was also attempted to puncture the vessel and dislodge the embolus into the vitreous cavity but was of no success. Retina surrounding the occluded artery was oedematous with oedema extending to the lower two-thirds of the macula. A partial cherry red spot was visible centrally with surrounding retina normally vascularized showing vessels of normal calibre and with a normal orange fundal glow [Figure 1 and 2]. Patient was sent for a detailed cardiology workup.

Complete physical examination by the cardiologist showed patient having a regular pulse but a loud first heart sound, a mid-diastolic murmur, and an absent carotid bruit. She was also checked thoroughly for any focal neurological deficit but was found to have none. Trans-thoracic echocardiography revealed the patient to have a thickened mitral valve with severe mitral valve 
stenosis of mitral valve area $0.8 \mathrm{~cm}^{2}$, mean trans-mitral pressure gradient of $10 \mathrm{mmHg}$ and moderate mitral regurgitation. Mitral valve stenosis is graded severe if mitral valve area is $<1.0 \mathrm{~cm}^{2}$ and trans-mitral pressure gradient of $\geq 10 \mathrm{mmHg}$ [1]. Sub-valvular strands of calcifications were seen which were considered a possible source of embolus [Figure 3 and 4]. She had a left ventricular ejection fraction of $60 \%$ with all other valves and structure normal. Same findings were also confirmed by trans-oesophageal echocardiography which also showed left atrial appendage to be free of thrombus.

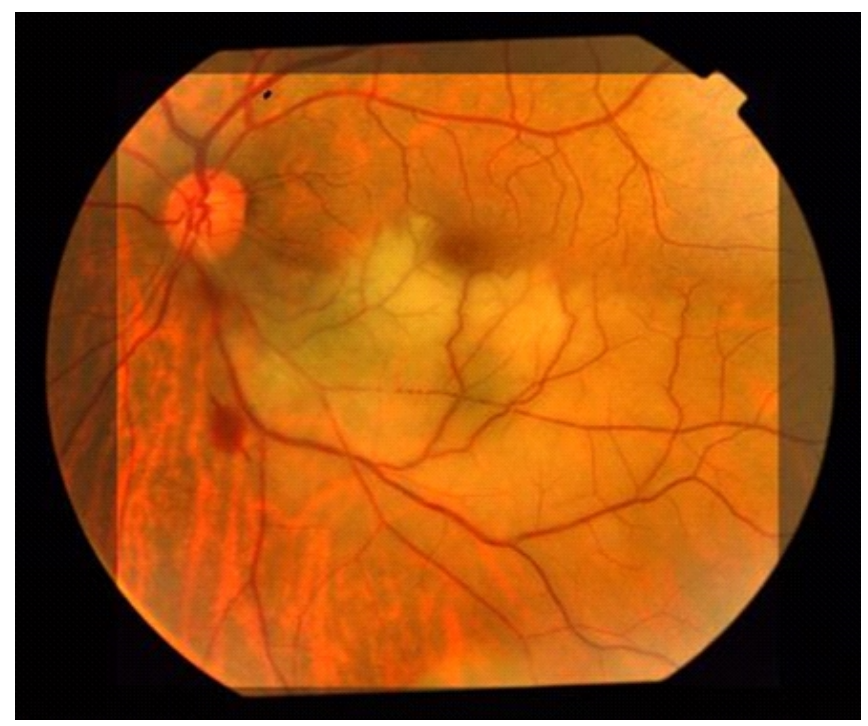

Figure 1. Fundal photograph showing lodged embolus in infero-temporal retinal artery with pale ischemic retina $\left(50^{\circ}\right.$ view).

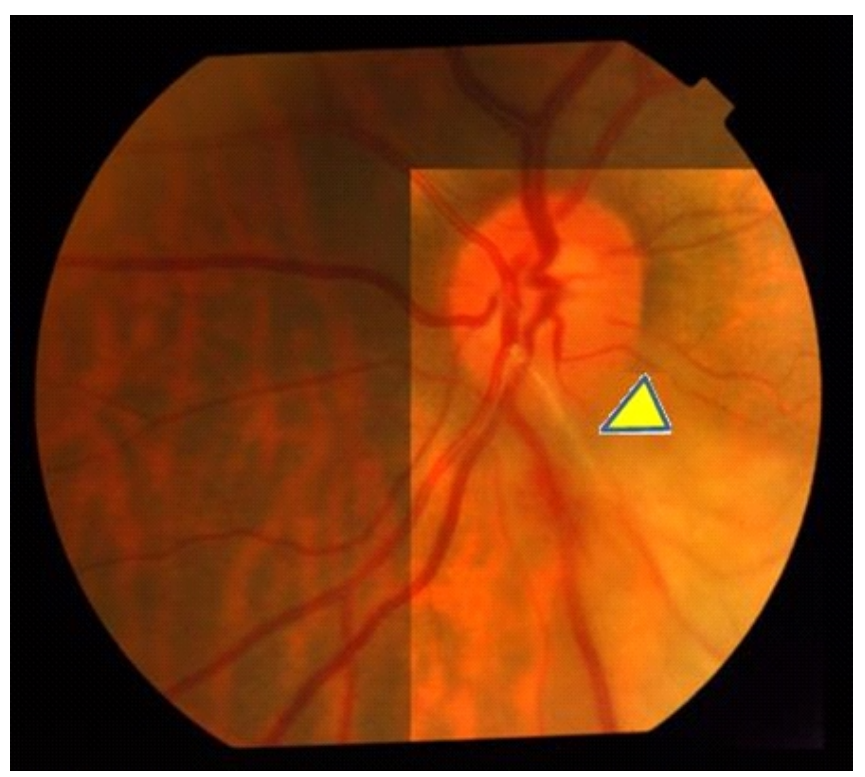

Figure 2. Fundal photograph showing lodged embolus (arrow) at higher magnification $\left(20^{\circ}\right.$ view)
Our patient didn't have atrial fibrillation and was in sinus rhythm. She was discharged on anti-coagulants with a definitive plan of mitral valve replacement since the valve was not suitable for percutaneous transvenous mitral commissurotomy because of calcification and moderate mitral regurgitation.

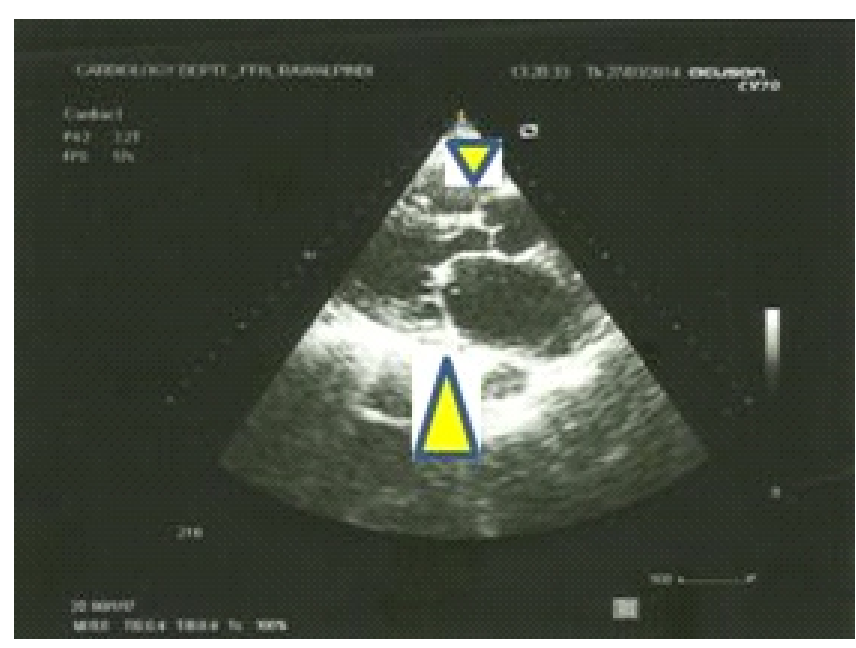

Figure 3. Parasternal long axis view. Large arrow, calcification of anterior mitral valve leaflet with restricted opening small arrow

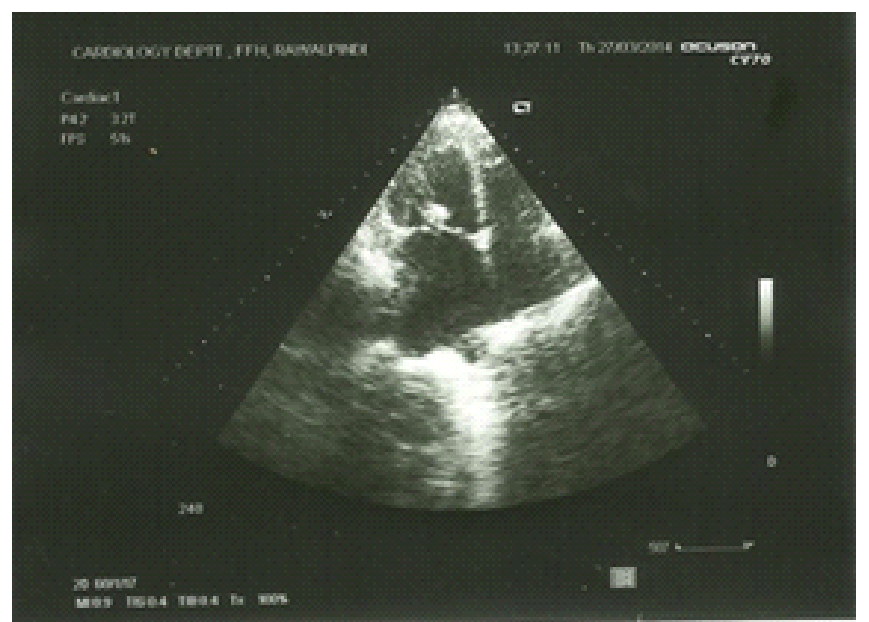

Figure 4. Apical four chamber view show dilated left atrium

\section{Discussion}

Most common cause of mitral valve stenosis is rheumatic fever. Rheumatic fever, on the other hand, involves the mitral valve most commonly as well. The onset of disease is mild with an insidious course. The symptoms only worsen when the dimensions of the left atrium increase and atrial fibrillation occurs which leads to thromboembolism. Thromboembolism most commonly occurs in the central nervous system followed by gangrene of the limb, myocardium and spleen. Thromboembolism in retinal vessels is a rare 
phenomenon. It is evident from the fact that CRAO, which constitutes for more than $50 \%$ of cases of retinal artery embolism, has an estimated incidence of $0.85 / 100,000 /$ year [9]. Another reported incidence of CRAO is 1 in 100,000 people with 1 visit in every 10,000 eye OPD visits [10]. Holley and colleagues have only reported 1 out of 165 patients with cardiac valvular disease having retinal embolism on autopsy [11].

The source of the thrombus from the carotid and due to essential thrombocythaemia were also excluded by carotid Doppler scan, biochemical tests, blood complete picture and bleeding time respectively. All of these tests showed normal results. Tests to exclude hypercoagulable states were not performed since the underlying diagnosis of mitral stenosis was obvious.

The fundal findings in our patient were consistent with fundal findings of other patients with embolised retinal arterial occlusion from cardiac valvular disease [12].

Patient was put on anti-coagulation therapy to prevent any chances of recurrent embolism. Medical therapy has failed in the past to control recurrent embolisms [13]. So a definitive management plan of mitral valve replacement was made. Another important indication of mitral valve replacement in our case was calcification in the valve as it has been previously shown to be highly associated with recurrent embolisms [14]. Mitral valve replacement with anti-coagulation as final treatment outcome has proven maximum success in preventing recurrent embolisms.

\section{Conclusion}

Mitral valve disease should be considered as a source of embolus in a patient presenting with retinal arterial occlusion. Regular fundal examinations should also be done in patients with mitral stenosis. Awareness of this possible but rare variant in presentation of mitral valve stenosis is beneficial for both ophthalmologist and cardiologists alike in rendering better management to such cases.

\section{References}

1. Ercan, S., Coskun, E., Altunbas, G., Oylumlu, M., Comez, A., Okumus, S., \& Davutoglu, V. (2013). Impact of Severe Mitral Stenosis on Visual Acuity, Intraocular Pressure, and Retinal Arteries. Int J Cardiovasc Res 2013, 2:6.

2. Davutoglu V, Soydinc S, Akdemir I, Turkmen S (2004) Paroxysmal Postural Dyspnea Related to Multiple Large Organized Thrombi in the Left Atrium. Clin Cardiol 27: 105.

3. Wiegand V, Tebbe U, Helmchen U, et al. Coronary arterial embolism due to valvular debris after percutaneous valvuloplasty of calcific mitral stenosis. Clin Cardiol 1988;11:793-6.

4. Winterkorn JM. Calcific retinal emboli and collateral shunting in a woman with rheumatic heart disease. Arch Ophthalmol 1995;113:1464-5.

5. Muci-Mendoza R, Parsa CF, Hoyt WF. Development of cilioretinal collaterals in a patient with calcific valvular heart disease. Arch Ophthalmol 1998;116:255.

6.Ragge NK, Hoyt WF. Nettleship collaterals: circumpapillary cilioretinal anastomoses after occlusion of the central retinal artery. $\mathrm{Br} J$ Ophthalmol 1992;76:186-8.

7. Brockmeier LB, Adolph RJ, Gustin BW, et al. Calcium emboli to the retinal artery in calcific aortic stenosis. $A m$ Heart J 1981;101:32-7.

8. Pantely GA, Housman LB, DeMots H, et al. Monocular blindness secondary to calcific embolization: an unusual presentation of rheumatic mitral valvular disease. Chest 1976;69:555-6.

9.Denniston AKO, Murray PI; Oxford Handbook of Ophthalmology (OUP), 2009

10.Rumelt S, Dorenboim Y, Rehany U. Aggressive systematic treatment for central retinal artery occlusion. Am J Ophthalmol. 1999;128:733-738.

11. Holley KE, Bahn RC, McGoon DC, et al. Spontaneous calcific embolization associated with calcific aortic stenosis. Circulation 1963;27:197-202.

12. Younge BR. The significance of retinal emboli. J Clin Neuroophthalmol 1989;9:190-4.

13. Brockmeier LB, Adolph RJ. Gustin BW: Calcium emboli to the retinal artery in calcific aortic stenosis (1981). Am Heart J101(1). 32-37.

14. Rancurel G, Marelle L, Vincent D, et al. Spontaneous calcific cerebral embolus from a calcific aortic stenosis in a middle cerebral artery infarct. Stroke 1989;20:691-3. 


\section{Key Points:}

- Pre existing mitral valve disease should be considered in cases presenting with retinal artery occlusion.

- Mitral valve disease can progress quietly, without producing any obvious clinical symptoms.

- Cases of mitral valve disease are at a risk factor for developing retinal artery occlusion. 